\title{
S-14: Soccer Injury Prevention Program; How Parents Can Play a Role?
}

\author{
Reza Rahimi Moghaddam, Maryam Asadi, Mohammad Haghshenas \\ Sports Medicine Association of Gilan Province, Iran
}

\begin{abstract}
INTRODUCTION: Soccer is classified as a high to moderate-intensity contact sport. It is therefore of importance that the incidence of soccer injuries be reduced through preventive interventions. The purpose of this review is to conclude the importance of a prevention program and explore the role parents have towards minimizing soccer related injuries among children and adolescence football players.

METHOD: 42 hand searches, 5 books, and 25 electronic articles were reviewed and relevant results were collected for the purpose of this paper. Selected studies were categorized as follows: soccer injury statistics, injury prevention program, and parents and prevention.

RESULTS: 5-16 year of age is a critical age range for soccer related injuries. Some studies have confirmed soccer injuries can be reduced by preventive interventions, and mentioned the importance of prevention program and the role of parents in the program. A few studies reported the efficacy for a positive parent-child relationship and injury prevalence, while other reported the negative influence parental demand on injury rates among children. Moreover, suggestions were made of consideration to parents prior to allowing children to participate in soccer.

CONCLUSIONS: Prevention of sports injuries is team work, and parent's role can be as vital as other members of the prevention team. In a successful preventive program, there are steps that parents can take to help kids stay safe on the soccer field or wherever they play or participate in sports activities. Educational materials should be provided to parents by soccer camp organizers before children involve in soccer programs.
\end{abstract}

Available at: http://journalofsportsmedicine.org and http://dx.doi.org/10.5152/tjsm.2017.018 